\title{
Bcl-2 antagonists and cancer: from the clinic, back to the bench
}

\author{
Cell Death and Disease (2010) 1, e37; doi:10.1038/cddis.2010.15; published online 29 April 2010 \\ Subject Category: Cancer
}

Manchester Cancer Research Centre Conference: Harnessing Apoptosis 17-20 January 2010, Manchester (UK)

This is an open-access article distributed under the terms of the Creative Commons Attribution License, which permits distribution and reproduction in any medium, provided the original author and source are credited. This license does not permit commercial exploitation without specific permission.

\section{Exciting News From the clinic: anticancer drugs acting on Bcl-2 proteins work!}

The Manchester Cancer Research Centre (MCRC), which is becoming the largest British center for clinical and preclinical research on cancer, recently organized a high profile conference. Cell death was the major theme of the meeting, which combined the contributions from world experts in cell biology and clinical translation of novel antitumour drugs that act on cell death pathways. For basic research scientists such as me, it was fascinating to learn the incredible advances that have been recently obtained in the clinical translation of new 'proapoptotic' drugs. It was also exciting to realize that so much research lies ahead for the clarification of cellular and biophysical aspects of these drugs, in particular for those acting on the Bcl-2 family of proteins that regulate cell death. Hence, successful clinical applications of drugs acting on $\mathrm{Bcl}-2$ proteins now stimulate renewed interests in the basic science of $\mathrm{Bcl}-2$ function, the perspectives of which I will outline in this meeting report.

\section{Discussion on how Bcl-2 proteins work together}

The mainframe model of how $\mathrm{Bcl}-2$ proteins regulate the key event in cell death signaling, namely the piercing of mitochondrial organelles (mitochondrial outer membrane permeabilization, MOMP), was established at the beginning of this century. Laboratories of the late Stanley Korsmeyer and of the ever-sparkling Doug Green, who delivered the opening lecture at the MCRC conference, contributed fundamental evidence supporting the model that has nowadays entered textbooks. However, as soon as this mainframe model was widely accepted, it generated a controversy that remains unsettled to date. The following question summarizes the controversy: Are proteins of the Bcl-2 family such as Bid and Bad equivalent in their proapoptotic capacity of inducing MOMP? Although various definitions have been used at the conference to describe the action of Bid and Bad, Bcl-2-binding drugs such as ABT-737 from Abbot Laboratories (Abbott Park, IL, USA) clearly act as antagonists, because they neutralize antiapoptotic proteins such as Bcl-2 itself. ABT-737 and its analogs were in fact designed to precisely mimic the $\mathrm{BH} 3$ domain of Bad, as elegantly presented by Saul Rosenberg at the conference. The $\mathrm{BH} 3$ domain corresponds to an amphipatic $\alpha$-helix representing the single region of sequence homology that is present in all the proteins of the Bcl-2 family and is essential for their reciprocal interaction. Bad is a typical 'BH3-only' protein because it contains only the $\mathrm{BH}$ domain of structural similarity. The diversity of Bad and other BH3-only proteins was discussed at the conference, but not enough to resolve the controversy on how Bcl-2 proteins work.

Although generally considered to be a $\mathrm{BH} 3$-only protein, Bid is in fact structurally and phylogenetically related to the multi-domain subfamily of the $\mathrm{Bcl}-2$ proteins, including all the antiapoptotic members and the gatekeepers of MOMP, Bax and its homologs. Of note, Bax and most multi-domain $\mathrm{Bcl}-2$ proteins share with Bid the property of interacting with membrane lipids and detergents. In contrast Bad, Bim, Puma, Noxa and other BH3-only proteins do not show common structural features with the multi-domain proteins of the family; therefore, they are $\mathrm{Bcl}-2$ analogs, rather than $\mathrm{Bcl}-2$ homologs. Indeed, they do not interact with membrane lipids and are also unrelated to the subfamily of BNIP3, an odd type of proapoptotic proteins seemingly associated with autophagic cell death. Autophagy is clearly involved in cancer and offers promising targets for new antitumour drugs, as presented by Eileen White at the conference.

Common structural and lipid-interacting properties may explain why only Bid, among the $\mathrm{BH} 3$-only proteins, is capable to catalytically activate Bax for inducing MOMP. Recent reports showing that the $\mathrm{BH} 3$ domains of Bim and Puma also elicit Bax activation may indicate additional reactions, which do not match the catalytic activation produced by Bid. Indeed, Bax binding of a stapled helix corresponding to the $\mathrm{BH} 3$ 
domain of Bim occurs at a site that is distant from the hydrophobic cleft that is involved in the $\mathrm{BH} 3-$ mediated interactions among $\mathrm{Bcl}-2$ proteins. This cleft forms the binding site of ABT-737 and other Bcl-2-interacting drugs, which mimic the action of $\mathrm{BH} 3-o n l y$ proteins and thus work as general antagonists of antiapoptotic Bcl-2 proteins. Bid constitutes an important exception to the generalization, as it can also activate Bax directly to promote the membrane perturbation leading to MOMP. By considering that Bax activation can occur also in the absence of other $\mathrm{Bcl}-2$ proteins, the details of this process remain unclear. More research is thus needed to clarify how Bax becomes activated, a central question in apoptosis research as indicated in the presentations of Doug Green and Tony Letai.

\section{Back to the bench: new approaches to clarify how Bcl-2 proteins and their drugs work}

The availability of clinically effective, small molecule BH3-mimetics such as ABT-737 will be exploited at the bench of biochemistry laboratories, as simplified model systems can effectively recapitulate the integrated action of $\mathrm{Bcl}-2$ proteins on biological membranes. Besides an increasing wealth of structural information, the study of Bcl-2 proteins has remained mainly based on changes in antibody reactivity, coupled to gel filtration and crosslinking approaches for detecting oligomers associated with Bax activation and MOMP. Membrane effects have been increasingly studied with liposomes mimicking the composition of mitochondrial membranes, which contain the unique lipid cardiolipin. Specific binding to cardiolipin has been documented for the caspasecleaved form of Bid (tBid) and appears to be fundamental for the integrated membrane action of tBid and Bax; it may also modulate the mitochondrial association of antiapoptotic $\mathrm{Bcl}-2$ proteins. However, cardiolipin may not be required for the proapoptotic action of $\mathrm{Bad}$, Bim and other $\mathrm{BH} 3-$ only proteins.

Most approaches focused on the aqueous phase do not produce accurate information on the protein dynamics at the surface of the membrane, where the activation and reciprocal interactions of $\mathrm{Bcl}-2$ proteins occur. These are the key reactions of $\mathrm{Bcl}-2$ proteins that we poorly understand, another problem that has emerged at the conference. The standard assay based upon the leakage of probes trapped within monolamellar liposomes cannot illuminate the details of how $\mathrm{Bcl}-2$ proteins dock onto the membrane surface and then penetrate the external lipid layer. Consequently, we hardly know how Bcl-2 proteins ultimately induce MOMP. On the other hand, assays normally used for measuring membrane fusion would be very appropriate for evaluating the rate-limiting steps in the process of membrane penetration by Bcl-2 proteins such as Bax. These assays can be developed by studying rapid changes in the fluorescence of lipid probes embedded within the external layer of liposomal membranes, an approach that I have pioneered with Bid. Additional model systems could be devised by using mixtures of aqueous and organic phases, such as inverted micelles, in which appropriately labeled Bcl-2 proteins could be monitored to penetrate and self-aggregate in the milieu of the lipid membrane. The outlined approaches could also elucidate the uncertainties regarding the cellular distribution of Bcl-2 antagonists such as ABT-737. ABT-737 and

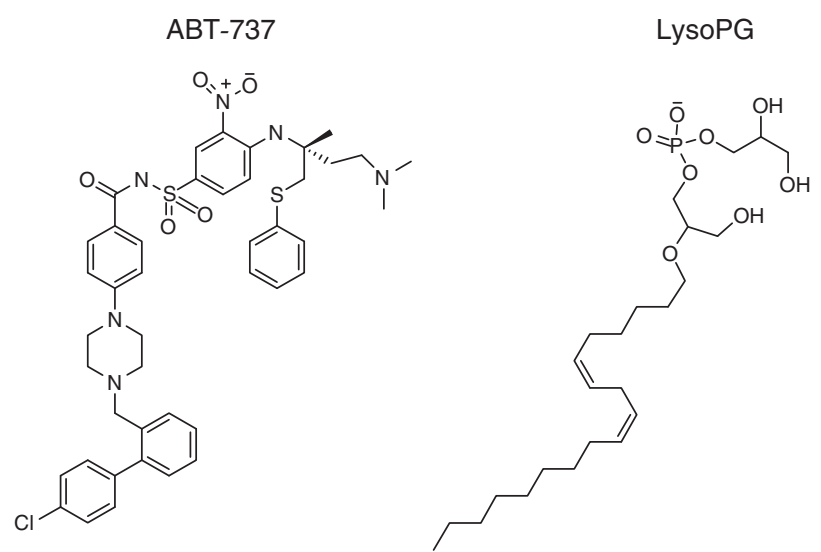

Figure 1 Structural comparison of the $\mathrm{Bcl}-2$ antagonist drug, $\mathrm{ABT}-737$, with a membrane lipid having detergent properties, 2-linoleyl-lyso-phosphatidyl-glycerol (LysoPG), which is related to the mitochondria-specific lipid cardiolipin modulating the action of Bcl-2 proteins

related drugs are large hydrophobic molecules that resemble in dimensions and some chemical properties like proportion of hydrophilic surface, detergent-like lipids related to cardiolipin, for instance lysophosphatidyl glycerol (Figure 1). They are thus likely to enter cells following the constitutive pathways of lipid traffic that are used by natural lysolipids. In this unexplored scenario, the drugs would then reach mitochondria after membrane fusion with vesicles from the endocytic and other compartments. Once incorporated within the outer mitochondrial membrane, how would the very lipophilic ABT-737 jump to the BH3-binding pocket of antiapoptotic Bcl-2 proteins, which normally protrudes in the aqueous phase of the cytosol? The new approaches suggested here will provide an experimental answer to this question, which was raised at the conference and has crucial pharmacological importance. Eventually, it will be possible to go back to the clinic with an advanced knowledge of the cellular and membrane-target distribution of $\mathrm{Bcl}-2$ antagonist drugs. The pertinent information, acquired by solid laboratory work of basic science, will be transferable to implement clinical applications. And then the learning cycle will be reversed: from the bench, back to the clinic! The theme for a future MCRC conference, perhaps.

\section{Conflict of interest}

The author declares no conflict of interest.

\section{Degli Esposti ${ }^{*, 1}$}

${ }^{1}$ Faculty of Life Sciences, University of Manchester, Oxford Road, Manchester M13 9PT, UK

*Corresponding author: M Degli Esposti, Faculty of Life Sciences, University of Manchester, Oxford Road, Manchester M13 9PT, UK. Tel: 44161275 5447; Fax: 44161275 5762; E-mail: mauro.esposti@manchester.ac.uk

(c) SOMERIEHIS RESERVED Derivative Works 3.0 License. To view a copy of this license, visit http:// creativecommons.org/licenses/by-nc-nd/3.0/ 